\title{
Video Article \\ Fabrication and Validation of an Organ-on-chip System with Integrated Electrodes to Directly Quantify Transendothelial Electrical Resistance
}

\author{
Marinke W. van der Helm ${ }^{1}$, Mathieu Odijk ${ }^{1}$, Jean-Philippe Frimat ${ }^{1,2}$, Andries D. van der Meer $^{3}$, Jan C.T. Eijkel ${ }^{1}$, Albert van den Berg ${ }^{1}$, Loes \\ I. Segerink ${ }^{1}$ \\ ${ }^{1}$ BIOS Lab on a Chip group, MIRA Institute for Biomedical Technology and Technical Medicine, MESA+ Institute for Nanotechnology and Max Planck Center for Complex \\ Fluid Dynamics, University of Twente \\ ${ }^{2}$ Microsystems, Eindhoven University of Technology \\ ${ }^{3}$ Applied Stem Cell Technologies, MIRA Institute for Biomedical Technology and Technical Medicine, University of Twente
}

Correspondence to: Marinke W. van der Helm at m.w.vanderhelm@utwente.nl

URL: https://www.jove.com/video/56334

DOI: doi:10.3791/56334

Keywords: Biomedical Engineering, Issue 127, Organs-on-chips, microfabrication, transendothelial electrical resistance, transepithelial electrical resistance, blood-brain barrier, microfluidics

Date Published: 9/26/2017

Citation: van der Helm, M.W., Odijk, M., Frimat, J.P., van der Meer, A.D., Eijkel, J.C., van den Berg, A., Segerink, L.I. Fabrication and Validation of an Organ-on-chip System with Integrated Electrodes to Directly Quantify Transendothelial Electrical Resistance. J. Vis. Exp. (127), e56334, doi:10.3791/56334 (2017).

\section{Abstract}

Organs-on-chips, in vitro models involving the culture of (human) tissues inside microfluidic devices, are rapidly emerging and promise to provide useful research tools for studying human health and disease. To characterize the barrier function of cell layers cultured inside organ-on-chip devices, often transendothelial or transepithelial electrical resistance (TEER) is measured. To this end, electrodes are usually integrated into the chip by micromachining methods to provide more stable measurements than is achieved with manual insertion of electrodes into the inlets of the chip. However, these electrodes frequently hamper visual inspection of the studied cell layer or require expensive cleanroom processes for fabrication. To overcome these limitations, the device described here contains four easily integrated electrodes that are placed and fixed outside of the culture area, making visual inspection possible. Using these four electrodes the resistance of six measurement paths can be quantified, from which the TEER can be directly isolated, independent of the resistance of culture medium-filled microchannels. The blood-brain barrier was replicated in this device and its TEER was monitored to show the device applicability. This chip, the integrated electrodes and the TEER determination method are generally applicable in organs-on-chips, both to mimic other organs or to be incorporated into existing organ-on-chip systems.

\section{Video Link}

The video component of this article can be found at https://www.jove.com/video/56334/

\section{Introduction}

Organs-on-chips are rapidly emerging as a new and promising class of in vitro tissue models. ${ }^{1}$ In these models, cells are cultured inside microfluidic devices that are engineered in such a way that they mimic the physiological microenvironment of those cells. ${ }^{1,2}$ This results in more realistic physiological or pathological behavior of those cells than can be expected from conventional in vitro models of simple design and basic function. ${ }^{3,5,6}$ In addition, organs-on-chips provide a better controllable environment than in vivo models and can incorporate both healthy and diseased tissue from human origin to faithfully replicate both human physiology and pathology. The recently summarized advances in the development of blood-brain barriers on chips (BBBs-on-chips) show that the field is quickly moving forward. ${ }^{7}$

Another advantage of organs-on-chips is that they enable real-time and continuous monitoring of the tissue cultured inside the device by microscopy, on-line biochemical analyses and integrated sensors. ${ }^{1,2}$ For example, measuring transendothelial or transepithelial electrical resistance (TEER) is a powerful method for monitoring non-invasively the development and disruption of barrier-forming tissues. ${ }^{8,9,10}$ TEER is the electrical resistance across a cellular barrier and is therefore indicative of the barrier integrity and permeability. ${ }^{10}$ In organs-on-chips, cellular barriers are generally cultured on a membrane that separates two fluidic channels, representing the apical and basolateral compartments of that barrier tissue. In such chips, TEER measurements can be conveniently conducted with electrodes inserted into the inlets and outlets of the two channels. ${ }^{3,4,11,12,13,14,15}$ However, manual insertion and reinsertion of the electrodes can easily result in placement errors and thus in variations in the measured resistance as e.g. the differences in resistance of longer or shorter paths through microchannels are significant compared to the cell barrier resistance. ${ }^{16}$ To eliminate reinsertion errors, devices with integrated electrodes have been proposed. However, most of these integrated electrodes block the view when inspecting the tissue culture ${ }^{17,18,19,20,21}$ and/or require specialized cleanroom processes for fabrication. ${ }^{17,22}$

The organ-on-chip device described in this publication, first applied in an earlier publication, ${ }^{16}$ combines the stability of integrated electrodes with visibility on the measured cell layer and easy fabrication. The design and fabrication of this chip is depicted in Figure 1. In short, this device 
consists of two polydimethylsiloxane (PDMS) parts with channel imprints that are bonded together leakage-free with a polycarbonate membrane with $0.4 \mu \mathrm{m}$ pores in between. Four platinum wire electrodes are inserted and fixed into place with a photocurable adhesive well outside of the culture area. All of these fabrication steps can be conducted with general laboratory equipment, without the need for a cleanroom environment. On top of this, six impedance measurements can be done using these four electrodes, thereby allowing direct isolation of the measured TEER, independent of the resistance of the microchannels leading up to the cross section and thus minimizing the influence of non-biological variations in the system such as (re)insertion errors. ${ }^{16}$

To show the applicability of this device and the direct TEER measurements, the blood-brain barrier (BBB) was replicated in this chip. This biological barrier consists of specialized endothelial cells and regulates transport between blood and brain to provide brain homeostasis. ${ }^{23,24}$ To mimic the BBB, the top channel of the microfluidic device was lined with human cerebral microvascular endothelial cells from the hCMEC/D3 cell line (kindly provided by Dr. P.-O. Couraud, INSERM, Paris, France). ${ }^{25}$ The presented method is, however, more generally applicable to any organ-on-chip device with two compartments, enabling direct TEER determination using easily integrated electrodes.

In this manuscript, first the fabrication process of the organ-on-chip device with integrated electrodes is described. Next, the seeding procedure and culture of brain endothelial cells inside the device is explained, as well as the on-chip TEER measurements. In the results section, representative TEER measurements are shown and data processing is clarified. Lastly, the barrier function of the BBB-on-chip, monitored during 3 days, is presented, showing the applicability of the presented device and methods to monitor TEER.

\section{Protocol}

\section{Fabrication of the organ-on-chip device}

1. Make a PDMS replica of a mold with the channel designs, fabricated using standard photolithography techniques, and obtain the PDMS parts of the microfluidic chip.

1. Weigh approximately $27 \mathrm{~g}$ PDMS base agent and $2.7 \mathrm{~g}$ curing agent; the mass ratio needs to be $10: 1$. This is good for a $3 \mathrm{~mm}$ thick PDMS slab on a mold with $130 \mathrm{~mm}$ (5 inch) diameter. Mix these components thoroughly.

2. Degas the mixture in a desiccator for approximately $45 \mathrm{~min}$ to remove air bubbles.

3. Meanwhile, prepare the mold for the liquid PDMS mixture by sticking clear tape around the mold or place the mold in a suitable wafer holder.

4. Pour the degassed PDMS mixture onto the mold. If any air bubbles remained in the PDMS mixture or at the mold surface, degas it again for approximately 30 min.

5. Cure the PDMS mixture in an oven at $60^{\circ} \mathrm{C}$ for $4 \mathrm{~h}$. Allow to cool down.

6. In a cross-flow hood, pull the cured PDMS from the mold; the mold can be reused immediately to make more PDMS replicas.

7. Cut the PDMS replica into separate top and bottom chip parts using cutting lines in the PDMS.

8. Punch four holes in the top parts using a sharp biopsy punch with $1 \mathrm{~mm}$ diameter to form inlets and outlets. Punch from inside to outside to prevent PDMS debris from collecting in the chip. Cover the chip parts with clear tape to protect them against dust.

9. Cut polycarbonate membranes from Transwell inserts into squares of approximately $3 \times 3 \mathrm{~mm}^{2}$.

2. Assemble a porous membrane leakage-free in between two PDMS parts in order to assemble a two-layer device interfaced with a porous membrane.

NOTE: This protocol is adapted from Griep et al. ${ }^{19}$ and Chueh et al. ${ }^{26}$

1. Prepare a PDMS/toluene mortar using $0.7 \mathrm{~g}$ of PDMS base agent, $0.07 \mathrm{~g}$ of curing agent and $540 \mu \mathrm{L}$ of toluene, resulting in a $5: 3$ weight ratio. Vortex the mortar thoroughly.

2. Spin-coat $200 \mu \mathrm{L}$ of mortar onto a glass coverslip at $1500 \mathrm{rpm}$ for $60 \mathrm{~s}$ (ramped at $1000 \mathrm{rpm} / \mathrm{s}$ ) to acquire a thin, uniform layer of mortar.

3. Transfer a thin layer of mortar from the coverslip onto a bottom part and a top part of the chip with an ink roller. Put the bottom part in an oven-safe dish.

4. With a set of tweezers, dip the edges of a membrane into the spin-coated mortar and place it carefully in the middle of the bottom part.

5. Carefully place the top part on the bottom part while paying attention to the alignment.

NOTE: Do not exert pressure onto the chip and do not slide the top part over the bottom part to prevent mortar from entering the channels and clogging the membrane.

6. Cover the chips inlets with clear tape to prevent dust from entering the chip and bake them at $60^{\circ} \mathrm{C}$ for $3 \mathrm{~h}$.

3. Integrate electrodes into the side channels.

NOTE: This protocol is adapted from Griep et al. ${ }^{19}$ and Douville et al..$^{18}$

1. Cut platinum wire into pieces of approximately $2 \mathrm{~cm}$. Clean by submerging them in acetone for 30 min. Rinse with water and ethanol and allow to dry.

2. In a cross-flow hood, put a chip on a plastic dish. Insert four platinum wires into the electrode channels of a chip using a pair of tweezers and bend them down onto the plastic dish to enable fixation to the dish in a subsequent step. Insert the wires $0.7-1 \mathrm{~mm}$ into the culture channel, past the T-shaped channel junction.

3. Apply a drop of UV-curable glue at the electrode channel entrance and allow the glue to fill the channel around the electrode by capillary forces.

4. Switch on UV and cure the glue when it reaches the end of the electrode channel.

Caution: Do not look into the UV light source as this may harm your eyes.

5. Fixate the four integrated electrodes to the plastic dish with a two-component epoxy adhesive. This allows easier handling of the electrodes during measurements without the risk of pulling the electrodes from the chip. 
6. Cover the chips with clear tape and bake them at $60{ }^{\circ} \mathrm{C}$ for $2 \mathrm{~h}$. Allow to cool down and store dust-free until use. In this way they can be safely stored for up to a month.

\section{On-chip culture of brain-derived endothelial cells}

1. Coat the chips to promote cell attachment.

1. Fill both channels with phosphate buffered saline (PBS) to wet them before introducing reagents. Check under a microscope if there are any air bubbles in the channels. If so, remove them by flushing with extra PBS.

2. Fill both channels with $30 \mu \mathrm{L}$ of $20 \mu \mathrm{g} / \mathrm{mL}$ human fibronectin in PBS. Incubate at $37^{\circ} \mathrm{C}$ for 3 hours. Check for air bubbles and, if needed, flush the channels with PBS and refill with fibronectin solution.

3. Flush the chips with endothelial growth medium and incubate them at $37{ }^{\circ} \mathrm{C}$ and $5 \% \mathrm{CO}_{2}$ in an incubator for $2 \mathrm{~h}$.

4. Measure the TEER of the blank chips as described in step 3 to ensure that all of the electrodes are in direct contact with fluid in the channels. In empty chips, typical TEER values are $0-1 \Omega \mathrm{cm}^{2}$.

2. Seed cells into the top channel.

1. Remove the culture medium from a culture flask $\left(75 \mathrm{~cm}^{2}\right.$ culture area) with a confluent monolayer of human cerebral microvascular endothelial cells (hCMEC/D3).

2. Wash the cells with PBS. Add $2 \mathrm{~mL}$ of $0.05 \%$ trypsin-EDTA and incubate at $37{ }^{\circ} \mathrm{C}$ and $5 \% \mathrm{CO}_{2}$ for $2-5$ minutes until the cells have detached from the culture flask.

3. Deactivate the trypsin with culture medium supplemented with $20 \%$ fetal bovine serum (FBS). Count the cells and calculate the total number of cells in suspension.

4. Meanwhile, centrifuge the hCMEC/D3 cells at $390 \times \mathrm{g}$ for $5 \mathrm{~min}$ and remove supernatant.

5. Resuspend the cell pellet in the appropriate volume of endothelial growth medium (EGM-2) to arrive at a concentration of $5 \times 10^{6}$ cells/ $\mathrm{mL}$. This results in a seeding density of $2 \times 10^{5}$ cells $/ \mathrm{cm}^{2}$ in the chip.

6. Slowly pipette $30 \mu \mathrm{L}$ of the well-mixed cell suspension into the top channel and remove the pipette from the inlet in a fluent motion while still exerting pressure.

7. Check the seeding density under a microscope. A uniform distribution of cells throughout the top channel should be achieved.

8. Incubate the chips at $37{ }^{\circ} \mathrm{C}$ and $5 \% \mathrm{CO}_{2}$ for at least $1 \mathrm{~h}$. Flush out any non-attached cells with endothelial culture medium.

3. Maintain the on-chip endothelial culture.

1. Twice per day, insert culture medium-filled pipette tips as reservoirs in the inlets and replace the medium inside the chip by gravitydriven flow.

NOTE: To avoid air bubbles from entering the microchannels and destroying the cell layer, make sure to make fluid-fluid contact between the pipette tip and the fluid on top of the chip before inserting the tip into an inlet. This can be facilitated by adding a smal drop at the in/outlet prior to pipette insertion.

2. Also add pipette tip reservoirs in the outlets to prevent the channels from drying.

3. Incubate the chips at $37^{\circ} \mathrm{C}$ and $5 \% \mathrm{CO}_{2}$.

\section{On-chip TEER measurements}

1. Set up the TEER measurement apparatus, consisting of a lock-in amplifier and a probe cable circuit as was specified in Van der Helm et al. ${ }^{16}$ Alternatively, potentiostats or impedance analyzers are also suitable for these impedance-based TEER measurements.

2. Take the chip from the incubator and allow it to reach room temperature for at least 10 min. Remove any fluid from the plastic dish around the electrodes to prevent electrode bridging outside of the chip.

3. Take the impedance spectrum from $200 \mathrm{~Hz}$ to $1 \mathrm{MHz}$ for each combination of two electrodes, resulting in 6 impedance spectra per chip in only 5-10 min. Check if the impedance spectra have the expected magnitudes and shapes to validate the TEER measurement, as is described in the results section.

4. Put the chip back in the incubator at $37{ }^{\circ} \mathrm{C}$ and $5 \% \mathrm{CO}_{2}$ after finishing the measurement.

5. Process the obtained impedance spectra to arrive at a normalized TEER value.

1. Get the measured resistance $R_{i \rightarrow j}$ between electrodes $i$ and $j$, as denoted in Figure $\mathbf{2 B}$ and $\mathbf{D}$, from the resistive plateau at $10 \mathrm{kHz}$ in the corresponding impedance spectra.

2. Calculate the TEER using the six measured resistances corresponding to one chip at one time point by using the equation of Figure $2 \mathbf{G}^{16}$ :

TEER $=A_{\text {cult }} \cdot R_{m}=A_{\text {cult }} \cdot \frac{1}{4}\left(R_{1 \rightarrow 2}+R_{1 \rightarrow 4}+R_{2 \rightarrow 3}+R_{3 \rightarrow 4}-2 R_{1 \rightarrow 3}-2 R_{2 \rightarrow 4}\right)\left[\Omega \mathrm{cm}^{2}\right]$

In this equation, $A_{\text {cult }}$ is the culture area through which the resistance was measured, $R_{m}$ is the resistance of the cellular barrier and the membrane, $R_{1 \rightarrow 2}, R_{1 \rightarrow 4}, R_{2 \rightarrow 3}$ and $R_{3 \rightarrow 4}$ are the resistance values measured through the cellular barrier and $R_{1 \rightarrow 3}$ and $R_{2 \rightarrow 4}$ are the resistance values measured in the straight channels. ${ }^{16}$ The equation is derived from the equivalent circuit illustrated in Figure 2C.

6. Subtract the blank TEER from the TEER at all time points to obtain the development of the TEER in time. 


\section{Representative Results}

The schematic results of electrical impedance spectroscopy through a chip without cells (solid line) and through a cellular barrier (dashed line) are shown in Figure 2A. Four main regions can be identified, each dominated by a specific electrical component. Below approximately $1 \mathrm{kHz}$, the double layer capacitance at the electrode-culture medium interface dominates, characterized by a negative slope for impedance magnitude and a phase shift approaching $-90^{\circ}$. The frequency at which the double layer capacitance dominates, depends on the electrode area exposed to culture medium. The resistive plateau above $1 \mathrm{kHz}$ (without cells) or $100 \mathrm{kHz}$ (with cells) with a phase shift close to $0^{\circ}$, corresponds to the resistance of the culture medium inside the microfluidic channels, depending on channel length and cross-sectional area, and inside the membrane, depending on the porosity and thickness. When measuring through a cellular barrier, an extra resistive plateau between 1 and 10 $\mathrm{kHz}$ is seen as well as a local maximum in the phase diagram. This region is of critical importance for the determination of the TEER as a clear increase in impedance results when cells are present in the measured path, and is therefore termed the "region of interest". The extra slope between 10 and $100 \mathrm{kHz}$ corresponds to the cell barrier capacitance, which arises from the electrically insulating lipid bilayer membranes and is dependent on the total area of the cell layer. ${ }^{27}$ The boundaries of these regions as well as the impedance magnitudes depend on the system being studied and change with, among other things, channel dimensions, culture medium conductivity, electrode position and cell type. For further reading about the theory and practice of electrical impedance spectroscopy on barrier-forming tissues the review article by Benson et al. is recommended. ${ }^{28}$

Representative data of the TEER measurements is shown for both a blank chip and a chip with a hCMEC/D3 brain endothelial cell layer in Figure 2E and 2F, respectively. In short, impedance spectroscopy was performed using six measurements with four electrodes: two measurements through cell culture medium-filled channels (solid lines) and four measurements through the channels as well as the membrane and - if present - the cellular barrier (dashed lines). These six measurement paths can be identified in the equivalent resistive circuit of Figure 2B, which is derived from the schematic cross section (Figure 2C) and top view (Figure 2D). The blank chip measurements (Figure 2E) show the typical shape of impedance spectra without cells, as illustrated in Figure 2A. The measurements through the cellular barrier (dashed lines in Figure 2F) resemble the typical impedance spectra with cells in Figure 2A. Note that both the impedance magnitude and the phase shift increase towards $1 \mathrm{MHz}$. This is the typical response of the measurement setup at high frequencies and is not of experimental origin.

To determine the TEER using these experimental impedance spectra, first the measured chip resistance is determined, which is the total resistance of the cell layer, channels and membrane. To this end, a suitable readout frequency in the region of interest was chosen, which is at the local maximum in the phase plot with cells and close to $0^{\circ}$ phase shift without cells: $10 \mathrm{kHz}$. With the six measured resistances at $10 \mathrm{kHz}$, as measured between the four electrodes, the TEER is directly calculated using the equation in Figure $\mathbf{2 F}$.

To show that the presented device is suitable for determining TEER in organs-on-chips, the BBB was replicated inside the chip and its TEER was monitored during 3 days of culture. In Figure 3A the average TEER \pm standard error of the mean (SEM) is shown for four BBBs-onchips, resulting in a plateau of $22 \pm 1.3 \Omega \mathrm{cm}^{2}$, which is comparable to the TEER of this cell line as reported in literature. ${ }^{29}$ Furthermore, after termination of the experiment and fluorescence staining of the nuclei, it can be seen that the brain endothelium formed a continuous monolayer inside the device (Figure 3B). Immunofluorescence staining of tight junction protein Zonula Occludens-1 (ZO-1) showed that the brain endothelial cells maintained their BBB-specific phenotype and formed tight junctional complexes.

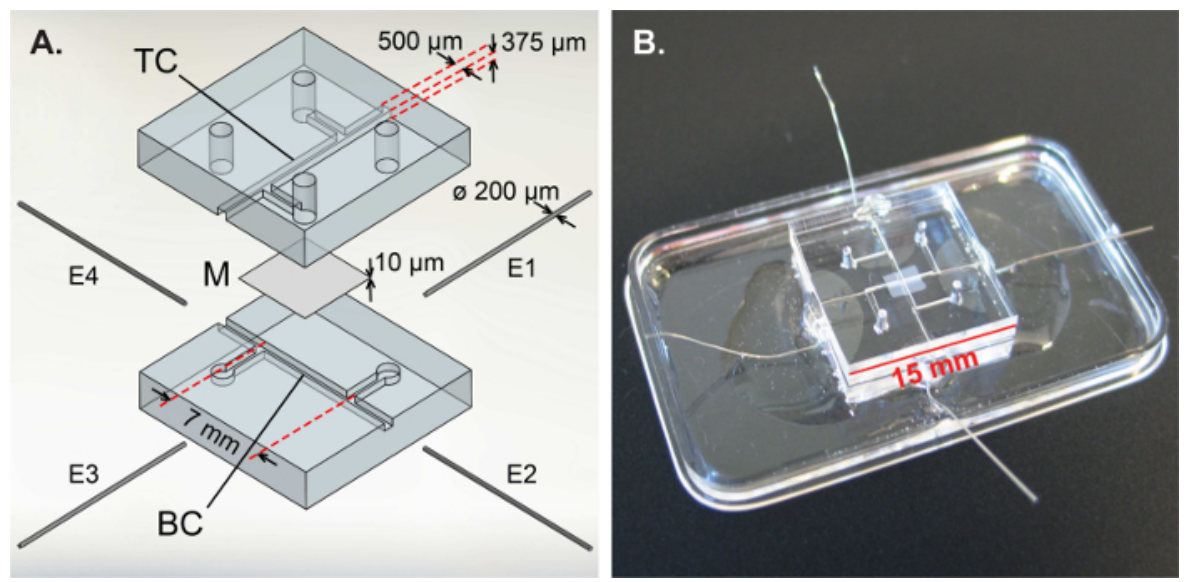

Figure 1: Design and assembly of the organ-on-chip device with integrated electrodes.

(A) Exploded view of the microfluidic chip, consisting of a top PDMS part with the top channel (TC), a membrane (M) and a bottom PDMS part with the bottom channel $(B C)$. Four platinum wire electrodes (E1-4) are inserted and fixed in the side channels. In the top channel and on top of the membrane, hCMEC/D3 brain endothelial cells were cultured to replicate the BBB. (B) Assembled chip, fixed to a plastic dish. Reprinted and adapted with permission from Elsevier. ${ }^{16}$ Please click here to view a larger version of this figure. 


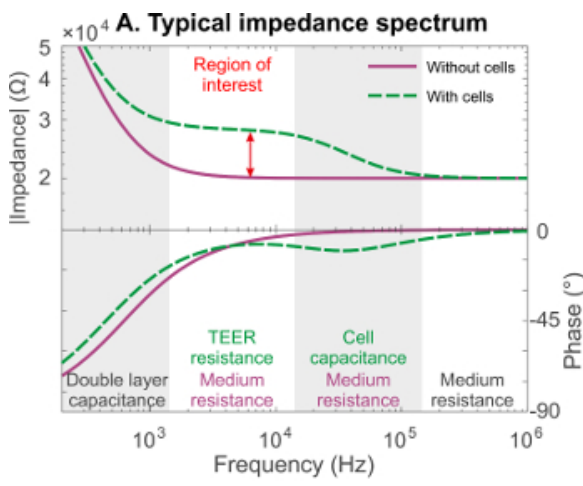

B. Equivalent resistive circuit

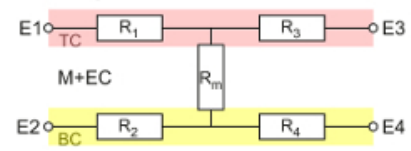

C. Schematic cross section
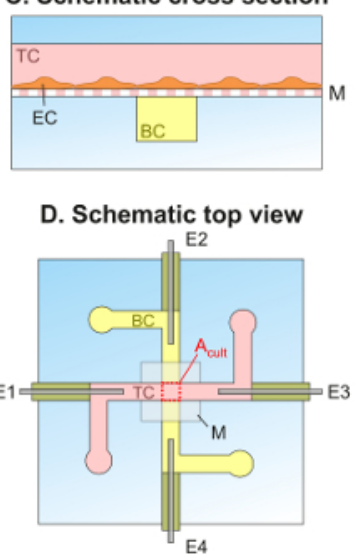

E. Blank chip
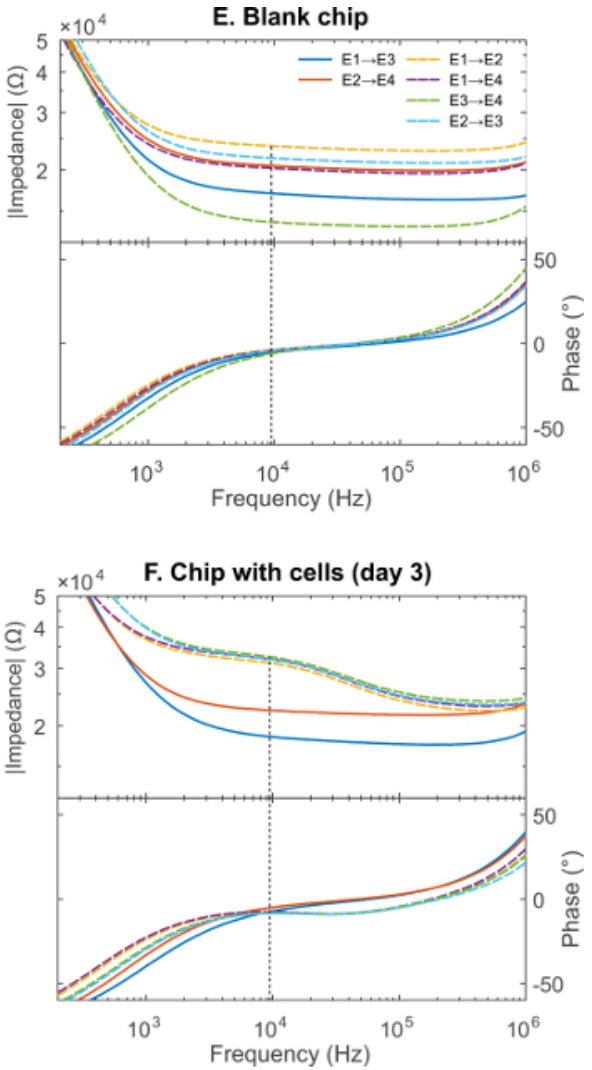

G. TEER quantification

TEER $=A_{\text {cult }} \cdot 1 / 4\left(R_{1 \rightarrow 2}+R_{1 \rightarrow 4}+\right.$

$\left.R_{2 \rightarrow 3}+R_{3 \rightarrow 4}-2 \cdot R_{1 \rightarrow 3}-2 \cdot R_{2-4}\right)$

Figure 2: Representative impedance data and determination of TEER.

(A) Schematic impedance spectrum showing impedance magnitude $(\Omega)$ and phase shift $\left({ }^{\circ}\right)$ versus frequency $(\mathrm{Hz})$, typical for electrical impedance spectroscopy on chips without cells (solid line) and with cells (dashed line). There are four main regions, each dominated by: the double layer capacitance at the electrodes, the culture medium resistance, the cell barrier resistance and the cell membrane capacitance. The "region of interest" indicates where the contribution of the cell layer can be quantified (red arrow). (B) Equivalent resistive circuit of the chip, showing the top channel resistors $R_{1}$ and $R_{3}$, the bottom channel resistors $R_{2}$ and $R_{4}$ and the membrane and $E C$ barrier resistor $R_{m}$. (C) Schematic cross section showing the endothelial cells (EC) cultured in the top channel. (D) Schematic top view of the BBB chip showing the configuration of the electrodes and the culture area of $0.25 \mathrm{~mm}^{2}$ through which the impedance is measured. (E) Representative impedance spectra of a blank chip filled with cell culture medium. (F) Representative impedance spectra of a chip in which hCMEC/D3 brain endothelial cells were culture for 3 days. (G) Formula to calculate TEER from the measured resistances between all six combinations of four electrodes. Reprinted and adapted with permission from Elsevier. ${ }^{16}$ Please click here to view a larger version of this figure. 


\section{A. TEER of BBBs-on-chips}

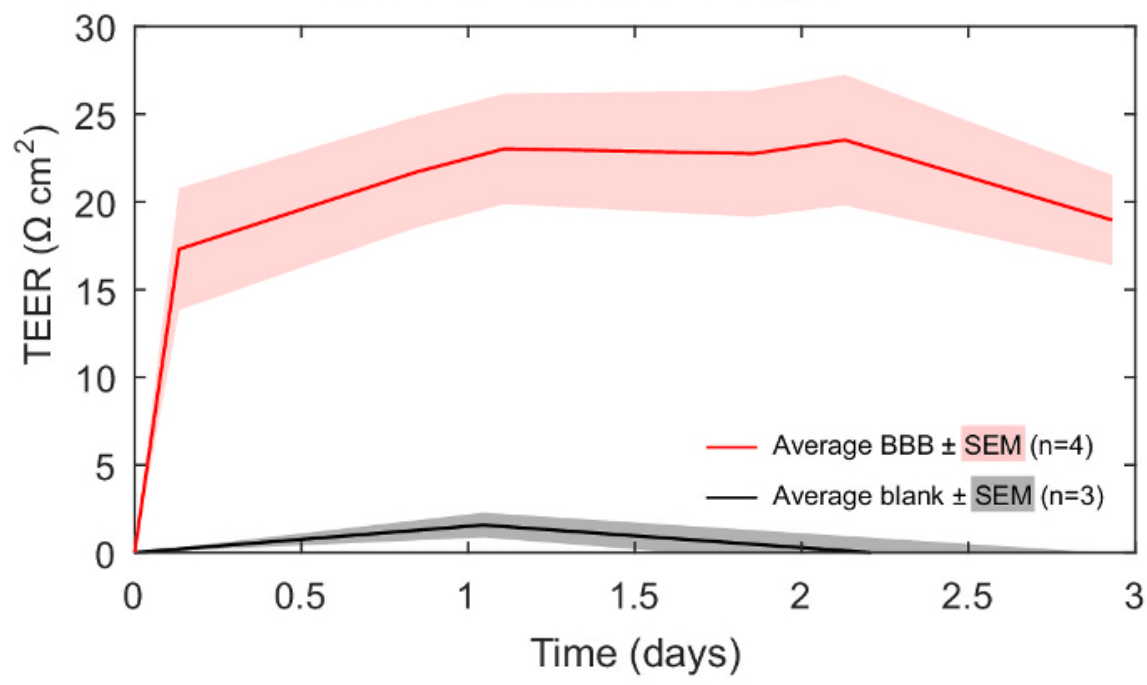

\section{B. Fluorescence microscopy of BBB-on-chip}

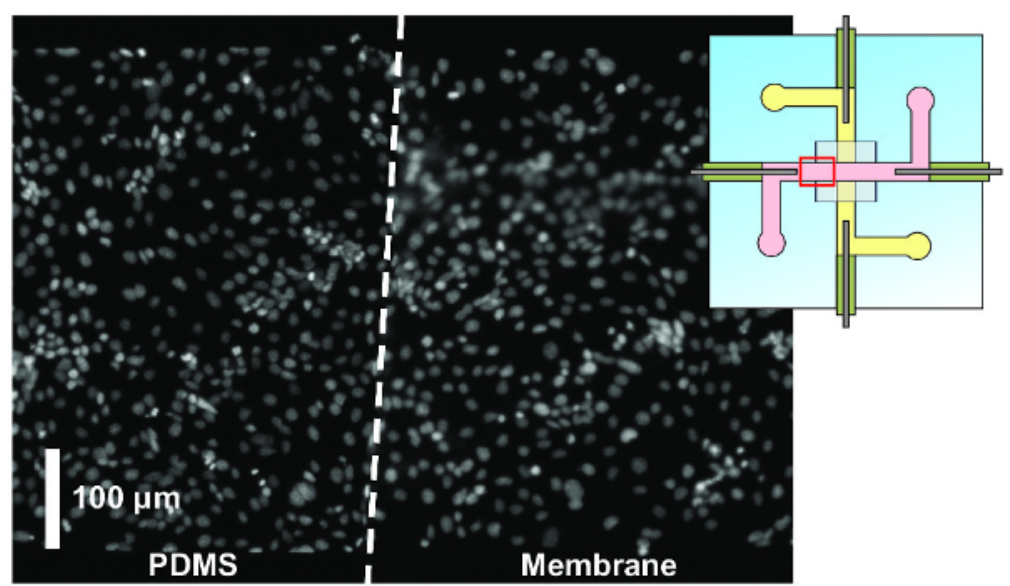

\section{Fluorescence microscopy of tight junctions}

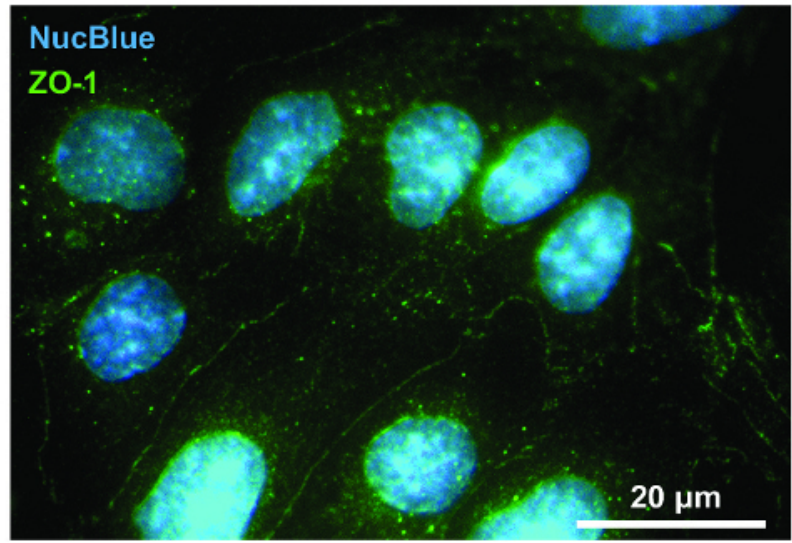

Figure 3: Representative TEER development of BBB-on-chip.

(A) Average TEER \pm standard error of the mean (SEM) of four BBBs-on-chips during a culture period of three days, reaching a plateau at 22 $\pm 1.3 \Omega \mathrm{cm}^{2}$ (average \pm SEM). For comparison, data of blank chips is included, showing marginal variation and deviation from $0 \Omega \mathrm{cm}^{2}$ in the same period compared to the variation and TEER value of chips with cells. (B) Fluorescence microscopy of stained nuclei revealed a continuous monolayer of endothelium, both on PDMS and the membrane at the location indicated in the inset. (C) Immunofluorescence revealed the presence of tight junction protein Zonula Occludens-1, indicating that BBB-specific tight junctions between the cells give rise to the measured TEER. Reprinted and adapted with permission from Elsevier. ${ }^{16}$ Please click here to view a larger version of this figure. 


\section{Discussion}

In this manuscript, the engineering of an organ-on-chip device and the direct determination of the transendothelial electrical resistance (TEER) of a cellular barrier cultured in the device were presented. The presented method of integrating electrodes without cleanroom equipment and the direct TEER determination using four electrodes is applicable to any organ-on-chip device with two microfluidic compartments. The chip layout and geometry can be adapted to fit the requirements of the envisioned experiments, as long as the four electrodes are separated in two compartments. The four electrodes can even be conveniently inserted in the inlets of existing chips, provided that they are fixated in place for the duration of the six measurements. The leakage-free bonding method can be optimized for different membranes and channel geometries by changing the PDMS/toluene ratio. A higher toluene content results in a thinner spin-coated layer of mortar ${ }^{26}$ and may be more suitable for shallower and narrower channels in the PDMS parts. A lower toluene content results in a thicker mortar layer ${ }^{26}$ and may be more suitable to enclose thicker membranes between the PDMS parts.

As can be seen in the schematic impedance spectra in Figure $2 \mathrm{~A}$ and the experimental spectra in Figure $\mathbf{2 E}$ and $2 \mathrm{~F}$, the impedance measurements are influenced by the double layer capacitance at the electrode-medium interface. Due to the small size of the electrodes inside the microchannels, the double layer capacitance can dominate over the resistive plateau of the cellular barrier in impedance spectra, complicating quantification of the TEER. To overcome this, the electrodes can be inserted further into the culture channels before fixation. This will increase the surface area of the electrode exposed to the culture medium and with that the double layer capacitance will increase as well. This results in a shift of the capacitive slope to lower frequencies, so that the resistive plateau of the cellular barrier can be more easily recognized and quantified. Although the resistance of the measured path between two electrodes will become smaller, this will not influence the TEER quantification following the presented method.

While measuring through the cellular barrier, it is possible that the extra resistive plateau cannot be recognized. This can be the result of a fluidic connection between the two channels, for example if the membrane is poorly enclosed by the mortar, leading to a measured path around the cellular barrier. In addition, there can be electrical bridging outside the chip if the electrodes are connected by a droplet of culture medium. This is generally combined with a lower measured impedance and can be solved by removing this bridge of culture medium. Lastly, if there is no resistive plateau and the measured impedance is orders of magnitude higher than expected, there may be a loose connection in the electrical wiring or at the power source.

In the future, the physiological relevance of the current BBB-on-chip can be increased by exposing the endothelial cells to shear stress at physiological levels, which is reported to promote BBB differentiation and increase barrier tightness and is hard to achieve in conventional in vitro models. ${ }^{29}$ In addition, the bottom channel of the presented device provides a suitable compartment for brain-derived cells to be co-cultured with the endothelium. This is also expected to increase the barrier function and also enables the study of the complex interactions between relevant cell types under pathological conditions. ${ }^{29}$

In conclusion, the organ-on-chip device described in this publication can be fabricated using standard laboratory equipment and is shown to provide direct TEER measurements using four integrated electrodes that do not impede visual inspection of the studied cell layer. The applicability of this device in the organs-on-chips field was demonstrated by mimicking the BBB in this chip and monitoring the TEER during the culture period. A host of other (barrier) organs can be mimicked by including other relevant cell types into this chip. In addition, the method for measuring TEER can be easily applied in other two compartment-based organ-on-chip devices to arrive at reproducible and meaningful TEER values that can be compared across devices and systems.

\section{Disclosures}

The authors have nothing to disclose.

\section{Acknowledgements}

We gratefully acknowledge Johan Bomer for fabrication of the mold and Mathijs Bronkhorst for fruitful discussions and assistance with data representation.

This research was funded by: SRO Biomedical Microdevices of L.I. Segerink, MIRA Institute for Biomedical Engineering and Technical Medicine, University of Twente; SRO Organs-on-Chips of A.D. van der Meer, MIRA Institute for Biomedical Engineering and Technical Medicine, University of Twente; and VESCEL, ERC Advanced Grant to A. van den Berg (grant no. 669768).

\section{References}

1. Bhatia, S. N., \& Ingber, D. E. Microfluidic organs-on-chips. Nat Biotechnol. 32 (8), 760-772 (2014)

2. Van der Meer, A. D., \& Van den Berg, A. Organs-on-chips: breaking the in vitro impasse. Integr Biol. 4 (5), $461-470$ (2012).

3. Huh, D. et al. Reconstituting organ-level lung functions on a chip. Science. 328 (5986), 1662-1668 (2010).

4. Kim, H. J., Huh, D., Hamilton, G., \& Ingber, D. E. Human gut-on-a-chip inhabited by microbial flora that experiences intestinal peristalsis-like motions and flow. Lab Chip. 12 (12), 2165-2174 (2012).

5. Kim, H. J., \& Ingber, D. E. Gut-on-a-Chip microenvironment induces human intestinal cells to undergo villus differentiation. Integr Biol. 5 (9), 1130-1140 (2013).

6. Westein, E. et al. Atherosclerotic geometries exacerbate pathological thrombus formation poststenosis in a von Willebrand factor-dependent manner. Proc Natl Acad Sci USA. 110 (4), 1357-1362 (2013). 
7. Van der Helm, M. W., Van der Meer, A. D., Eijkel, J. C., Van den Berg, A., \& Segerink, L. I. Microfluidic organ-on-chip technology for bloodbrain barrier research. Tissue barriers. 4 (1), e1142493 (2016).

8. Abbott, N. J., Dolman, D. M., Yusof, S., \& Reichel, A. in Drug Delivery to the Brain Vol. 10 AAPS Advances in the Pharmaceutical Sciences Series. (eds Margareta Hammarlund-Udenaes, Elizabeth C. M. de Lange, \& Robert G. Thorne) Ch. 6, 163-197 Springer New York. (2014).

9. Odijk, M. et al. Measuring direct current trans-epithelial electrical resistance in organ-on-a-chip microsystems. Lab Chip. 15 (3), 745-752 (2015).

10. Srinivasan, B. et al. TEER Measurement Techniques for In Vitro Barrier Model Systems. J Lab Autom. 20 (2), $107-126$ (2015).

11. Brown, J. A. et al. Recreating blood-brain barrier physiology and structure on chip: A novel neurovascular microfluidic bioreactor. Biomicrofluidics. 9 (5), 054124 (2015).

12. Deosarkar, S. P. et al. A Novel Dynamic Neonatal Blood-Brain Barrier on a Chip. PLoS ONE. 10 (11), e0142725 (2015).

13. Ferrell, N. et al. A microfluidic bioreactor with integrated transepithelial electrical resistance (TEER) measurement electrodes for evaluation of renal epithelial cells. Biotechnol bioeng. 107 (4), 707-716 (2010).

14. Kilic, O. et al. Brain-on-a-chip model enables analysis of human neuronal differentiation and chemotaxis. Lab Chip. 16 (21), $4152-4162$ (2016).

15. Partyka, P. P. et al. Mechanical stress regulates transport in a compliant 3D model of the blood-brain barrier. Biomaterials. 115 30-39 (2017).

16. Van der Helm, M. W. et al. Direct quantification of transendothelial electrical resistance in organs-on-chips. Biosens Bioelectr. 85 924-929 (2016).

17. Booth, R., \& Kim, H. Characterization of a microfluidic in vitro model of the blood-brain barrier (mu BBB). Lab Chip. 12 (10), 1784-1792 (2012).

18. Douville, N. J. et al. Fabrication of two-layered channel system with embedded electrodes to measure resistance across epithelial and endothelial barriers. Anal chemi. 82 (6), 2505-2511 (2010).

19. Griep, L. M. et al. BBB ON CHIP: microfluidic platform to mechanically and biochemically modulate blood-brain barrier function. Biomed Microdevices. 15 (1), 145-150 (2013).

20. Wang, Y. I., Abaci, H. E., \& Shuler, M. L. Microfluidic blood-brain barrier model provides in vivo-like barrier properties for drug permeability screening. Biotechnol Bioeng. (2016).

21. Wang, J. D., Khafagy, E.-S., Khanafer, K., Takayama, S., \& El Sayed, M. E. H. Organization of Endothelial Cells, Pericytes, and Astrocytes into a 3D Microfluidic in Vitro Model of the Blood-Brain Barrier. Mol Pharm. 13 (3), 895-906 (2016).

22. Walter, F. R. et al. A versatile lab-on-a-chip tool for modeling biological barriers. Sens Actuators B Chem. 222 1209-1219 (2016).

23. Abbott, N. J., Patabendige, A. A., Dolman, D. E., Yusof, S. R., \& Begley, D. J. Structure and function of the blood-brain barrier. Neurobiol Dis. 37 (1), 13-25 (2010).

24. Cardoso, F. L., Brites, D., \& Brito, M. A. Looking at the blood-brain barrier: molecular anatomy and possible investigation approaches. Brain Res Rev. 64 (2), 328-363 (2010).

25. Weksler, B. et al. Blood-brain barrier-specific properties of a human adult brain endothelial cell line. FASEB J. 19 (13), 1872-1874 (2005).

26. Chueh, B.-H. et al. Leakage-free bonding of porous membranes into layered microfluidic array systems. Anal chem. 79 (9), $3504-3508$ (2007).

27. Golowasch, J., \& Nadim, F. in Encyclopedia of Computational Neuroscience. (eds Dieter Jaeger \& Ranu Jung) 555-558. Springer New York. (2015).

28. Benson, K., Cramer, S., \& Galla, H.-J. Impedance-based cell monitoring: barrier properties and beyond. Fluids Barriers CNS. 10 (5) (2013).

29. Weksler, B., Romero, I. A., \& Couraud, P.-O. The hCMEC/D3 cell line as a model of the human blood brain barrier. Fluids Barriers CNS. 10 (1), 16 (2013). 